Monatsschrift für Geburtshülfe und Gynaekologie 1898;7:597-599

\title{
Tagesnachrichten und Notizen
}

Hofrat Dr. Osterloh ist zum Oberarzt der neubegriindeten Ab-teilung fiir Frauenkrankheiten am Dresdner Stadtkrankenhaus ernannt worden. Dieselbe wird ungefähr 40 Betten umfassen. Dr. Osterloh ist Schüler Credo's und v. Winckel's, war seit 1882 als Gynäkologe am

Diakonissenliaus in Dresden thätig, wo auf seinen Antrag bei dem Neubau desselben eine rein gynäkologische Abteilung gegriindet und unter seiner oberärztlichen Leitung im Oktober 1894 eröffnet wurde. Auch das Carola-haus in Dresden besitzt eine eigene gynäkologische Abteilung mit Dr. Justus Schramm als Oberarzt. Unseres Wissens ist dies der erste 598

Tagesnachrichten und Notizen.

Fall in Deutschland, dass ein grosses Stadtkrankenhaus eine besondere Ab-teilung fur Gynäkologie errichtete und mit einen Oberarzt besetzte. Die gynäkologischen Fälle sind bis jetzt selbst in grossen Krankenhäusern, teils in der inneren, teils in der chirurgischen Abteilung untergebraclit. Die unerlässlichsten Operationen werden gewöhnlich von dem Chirurgen ausgeführt, der nur ausnalimsweise auch in Gynäkologie ausgebildet ist, während die auf der inneren Abteilung verpflegten gynäkologischen Fälle aus gleicher Ursache sehr oft nur eine unzulängíiche gynäkologisclie Fach-behancllung erfahren. Durch Anstellung von Chirurgen, welche auch eine gewisse gynäkologisclie Ausbildung nachweisen, wird dem TJbelstand einer oft mangelhaften fachlichen Behandlung gynäkologischer Fälle nicht be-friedigend abgeholfen, da schliesslich doch die chirurgische Thätigkeit über-wiegen wird. Bei der Selbständigkeit und der hohen Entwickelung der modernen Gynäkologie als einer besonderen Fachdisziplin erschoint darum, zunächstfür grössere Krankenhäuser, die Schaffung besonderer gynäkologischer Abteilungen, allenfalls in Verbindung mit einer geburtshilflichen Abzweigung, unter eigenen Oberärzten als eine von der Gynäkologie mit Recht zu erhebende Forderung, der, wie nach dem Beispiele Dresdens gehofft werden darf, in Zukunft mehr und mehr entsprochen werden wird, auch zur Ent-lastung der bestehenden Krankenabteilungen und zum Wohle der Kranken.

Dr. J. Backer, Schüler von Prof, von Késmárszky, hat sich für Geburtshülfe und Gynäkologie an der Universität Budapest habilitirt.

Dr. Tullio Rossi-Doria in Rom und Dr. S. Patellani in Parma haben sich für Geburtshülfe und Gynäkologie habilitiert.

Prof. Werth in Kiel ist zum Geheimen Medizinalrath ernannt worden.

Die No. 11 des ,,Progrès Medical” vom 12. März 1898 enthält eine ausführliche Biographie des Nachfolgers von Tarnier auf dem Lehrstuhl der Geburtshülfe an der Pariser medizinischen Fakultät, Pierre Budin, auf welche wir bei dem hohen Ansehen, das dieser auch bei uns geniesst, gerne vei-weisen.

Von dem „Allgemeinen Yerein für Verbesserung der Frauenkleidung” werden wir ersucht, die beifolgende Notiz aufzunehmen. Ob-wohl das uns zugesandte Cirkular keineriei

Namensunterschrift aufweist, haben wir urn des guten Zweckes willen den Abdruck nicht abgelehnt. Es lautet:

Allgemeiner Verein für Verbesserung der Frauenkleidung. 
(Ausstellung für verbesserte Frauenkleidung und für Frauenhygiene.)

Der Allgemeine Verein für Verbesserung der Frauenkleidung, Zweig-verein Berlin, veranstaltet Ende September d. J. eine umfangreiche Ausstellung für verbesserte Frauenkleidung und für Frauenhygiene. Der Vor-stand des Vereins fühlt sich durch das grosse Interesse, das die im Arorigen Jahre hierselbst und in diesem Jalire in Dresden und Frankfurt a. M. statt-gefundenen gleichen Ausstellungen, trotz ihres beschrränkten Umfanges, entgegengebracht $\Lambda$ vurde, veranlasst, eine Ausstellung zu veranstalten, die möglichst ein Gesamtbild der Leistungen auf diesem Gebiete alien Inter-essenten vor Augen führen soil und gleichzeitig durch die Yielseitigkeit, so $\lambda$ vie durch die Art der Gegenstände selbst, den Beweis erbringen soil, wie sehr die vom Yereine theoretisch angeregten Verbesserungen, trotz der kurzen Zeit des Bestehens des Vereines, sich in vielseitiger Beziehung praktisch verbreitet und eingeführt haben. Das Ausstellungsbureau be-findet sich in der Geschäftsstelle des genannten Vereins, Potsdamerstrasse 121 G. (9-1.)

Litteratu $\Gamma$-Verzeiclmiss. $\quad 599$

Beriehtig ung .

In dem Aufsatze von Worms er, Die Kolpektomie zur Beseiti-gung des Prolapses älterer Frauen, Monatssclirift, Bd. VII, Heft

4, ist em sinnentstellender Druckfehler übersehen worden. Auf S. 372,

5. Zeile $\tau$ on unten muss es heissen: ..in keinem Falle wurde eine Hydro-

metra konstatiert*, anstatt ,in eineni Falle”.

Litteratur-Verzeichniss.

I. Geburtshilfe.

1. Bücher. Monograph iem Beri elite.

Coscntwo, Gr·, Rendiconto della clinica ostetrico-ginecologica pareggiata

dell'ospedale S. Francesco Saverio. Arch, di Ost. e Gin. Anno V.

No. 3. 1898. Gl·ottsch <> lk, S., Ueber den Einfluss des Wochenbettes auf cystische Eierstocksgescli $\Lambda$ vtilste. Sammlung k1. Vortr. N. F. No. 207. Leipzig,

Breitkopf u. Härtel. 1898. Grosse, Johannes, Ignaz Philipp Semmelweis, der Entdecker der

Ursache

des Kindbett-Fiebers. Leipzig- $\Lambda$ Vien. Deuticke. 1898. Kollmann, J., Lehrbuch der

Entwicklungsgeschichte des Menschen. Jena,

Gustav Fischer, 1898.

2. Allgemeines.

Bachimont, Francois, Documents pour servir a l'histoire de la puériculture

iutra-utérine. These de Paris. 1898. E > ieher, Zur Verminderung der Geburtenzahl. Deutsche Mecl. Wochschr.

No. 14. 1898. DßCÏo, Cenno storieo-critico-confermativo della priorità di una peculiare giacitura delle partorienti. Ann. di Ost. e Gin. Num. 3. · 1898. Deppisch, 1st die Tötung der lebenskräf $1 / 8$ en Frucht im Beckenausgang zu-

lässig? Munch. Med. Wochschr. No. 11. 1898. MeL·lar, C. Derde serie ran 200 accouchementen.

Nederl. Tiids. voor

Yerlosk. en Gym Afl. 1. 1898.

3. Physiologie und Pathologie der S divan gersch aft, der Geburt, des $\Lambda$ Vochenbetts.

Eonnemaison, Des accouchements rapides et non surveillés et de leurs complications. These de Paris. 1898. 
Eonnaire, Goitre et grossesse, auto-intoxication thyroïdienne. Bull, de la Soc. d'Obst. de Paris. No. 1. 1898.

Brünings , 'III. , Tetanus uteri und drohende Uterusruptur. Centralbl. für Gym No. 15, 1898.

Delage, De la choree gravidique. These de Paris. 1898.

Delbanco, Ein Fall von tuberösem subchorialeu Haematom der Decidua. Munch. Med.

Wochschr. No. 13. 1898.

Jacobs, C, La laparotomie dans certains cas de rétroposition pathologique de l'utérus pendant la grossesse. Journ. d'Accouchem. No. 15. 1898.

Lantos, E-, Ilvdramnios bei Drillingsschwangerschaft. Centralbl. für Gym No. 11. 1898.

TjOrain, Fibromes et accouchement. La Rev. Mèd. No. 192. 1898. 